\title{
La FMH salue la nouvelle Loi sur les professions de la psychologie
}

\section{Christine Romann}

Membre du Comité central de la FMH, Responsable du domaine Promotion de la santé et prévention
Correspondance:

Dr Christine Romann

FMH

Elfenstrasse 18

$\mathrm{CH}-3000$ Berne 15

Tél. 0313591111

Fax 0313591112

christine.romann@bluewin.ch
Toute personne qui fait appel à un ou une psychologue aimerait être sûre qu'il ou elle se confie à quelqu'un de compétent. Cela s'applique d'autant plus au domaine délicat de la psychothérapie psychologique dispensée par des psychologues qui, comme d'ailleurs l'ensemble des professions de la psychologie, attendent depuis longtemps l'instauration d'une législation en la matière. En 1991 déjà, la Conférence suisse des directrices et directeurs cantonaux de la santé demandait au Conseil fédéral de réglementer la formation prégraduée, postgraduée et continue des psychothérapeutes, à l'époque cette réglementation était encore prévue dans le cadre des professions médicales. Ce projet a essuyé de violentes critiques et, en 1998, le Conseil fédéral mandata le Département fédéral de l'intérieur pour élaborer une loi sur les professions relevant du domaine de la psychologie. En 2001, le Parlement se rallia au projet en approuvant la proposition parlementaire s'y rapportant. Et finalement en 2005, une première ébauche de la loi actuelle a été envoyée en consultation où elle suscita à nouveau de nombreuses critiques. Suite à un remaniement complet, le projet de loi a été encore une fois soumis en février 2009 à un cercle d'intéressés. Cette loi retravaillée à plusieurs reprises est actuellement en discussion aux Chambres fédérales.

L'objectif de la Loi sur les professions de la psychologie est la protection de la santé psychique: les personnes qui recourent à des prestations dans ce domaine ne doivent pas pouvoir être induites en erreur par leur psychologue. A cette fin le Conseil fédéral veut clairement déterminer les professions et des titres postgrades fédéraux protégés. La loi prévoit que seules les personnes au bénéfice d'un master en psychologie pourront faire usage de la dénomination de psychologue et proposer des prestations dans ce domaine. La psychothérapie psychologique est réglée de manière plus approfondie: pour pouvoir accomplir une formation postgraduée en psychothérapie, le candidat devra être titulaire d'un master en psychologie, et la «psychologie clinique» incluse dans ce cursus de formation. De plus, la filière de formation en psychothérapie psychologique doit être accréditée sur le plan fédéral. Le projet de loi prévoit cependant une certaine souplesse en ce qui concerne le cursus universitaire: les détenteurs d'un titre universitaire apparenté à la psychologie doivent pouvoir accéder à une formation en psychologie à des conditions appropriées. La FMH est favorable à une solution plus large à savoir que toute personne disposant d'un Bachelor dans une discipline apparentée à la psychologie, telle que la pédagogie p. ex. , doit pouvoir être admise à un master en psychologie.

Dans l'ensemble, le projet de loi est convaincant et fidèle à l'objectif visé. La protection en matière de titres crée une plus grande transparence pour les consommateurs. L'introduction d'une formation postgraduée accréditée en psychothérapie psychologique vise la haute qualité qui est nécessaire dans un domaine aussi délicat que la psychothérapie psychologique.

\section{Chambre médicale - élection de deux membres du Comité central}

La Dr Marie-Christine Peter et le Dr Max Giger, tout deux membres du Comité central, se retireront de leurs fonctions le $\mathbf{2 7}$ mai 2010 après une activité longue et fructueuse.

La Chambre médicale ordinaire du $\mathbf{2 7}$ mai 2010 devra donc se consacrer à leur succession et à l'élection de deux nouveaux membres du Comité central.

Nous aimerions offrir aux candidats la possibilité de se présenter préalablement dans les colonnes du BMS. Pour de plus amples informations à ce sujet, veuillez adresser un courriel au service de la communication de la FMH (kommunikation@ fmh.ch). Sur demande, nous vous transmettrons un document précisant les informations requises ainsi que des exemples de portraits déjà publiés. Vous voudrez bien renvoyer vos informations d'ici au 19 avril 2010 au plus tard. Les portraits des candidats paraîtront dans le Bulletin des médecins suisses du 12 mai 2010.

Service de communication de la FMH 